\title{
Billroth II gastrektomili hastalarda koledok taşlarının temizlenmesi için kısmi biliyer sfinkterotomi ile birlikte "large" balon dilatasyonun etkinliği
}

\author{
Efficacy of limited endoscopic sphincterotomy with endoscopic papillary large balloon dilatation in \\ Billroth II gastrectomy patients with bile duct stones
}

Selçuk DIŞIBEYAZ, Erkan PARLAK, Bülent ÖDEMIŞ, Diğdem ÖZER ETIK, Olga METIN, Semih SEZER, Nurgül ŞAŞMAZ, Burhan ŞAHIN

Türkiye Yüksek Ihtisas Hastanesi, Gastroenteroloji Kliniği, Ankara

\begin{abstract}
Amaç: Billroth-II gastrektomi ameliyatı geçirmiş olan hastalarda değişen anatomik özellikler nedeniyle safra yolu taşlarının çıkartılması güçlük arz eder. Son yıllarda özellikle zor safra taşlarının çıkartılmasında endoskopik sfinkterotomiden sonra endoskopik papiller "large" ballon dilatasyonu uygulanmaktadır. Bu çalışmanın amacı Billroth-II ameliyatı geçirmiş olan hastalarda endoskopik sfinkterotomi sonrası endoskopik papiller "large" ballon dilatasyonunun safra yolu taşı temizlenmesinde etkinliğinin ve güvenirliğinin araştırılmasıdır. Gereç ve Yöntem: Billroth-II ameliyatı geçirmiş, safra yolu taşı olan ve konvansiyonel endoskopik sfinkterotomi ile taşı çıkartılamayacağı düşünülen hastalarda endoskopik sfinkterotomiyi takiben, koledok çapına eşit, 12-18 mm 'large" ballon dilatasyonu uyguland1. Taşlar balon, basket veya mekanik litotripsi ile çıkartıldı. Bu yöntemin başarı ve komplikasyon oranları incelendi. Bulgular: On iki [hepsi erkek, ortalama yas 69.0 (aralık 48-84) yıl)] Billroth-II ameliyatı geçirmiş olan hastaya kısmi endoskopik sfinkterotomiyi takiben 'large" ballon dilatasyonu ile taş çıkarma girişiminde bulunuldu. 6 hastaya $12 \mathrm{~mm}, 6$ hastaya $15 \mathrm{~mm}, 1$ hastaya $18 \mathrm{~mm}$ balon uygulandı. Taş boyutu (enine çapı) ortalama 14,6 (aralık 12-18) mm idi. Koledok çapı ortalama 15,8 (arahı 10-21) mm idi. On bir hastanın taşları 1 seansta, 1 hastanın taşları 2 seansta çıkartıldı. 1 hastaya mekanik litotripsi, I hastaya ESWL yardımı gerekti. Işlemden sonra 1 hastada az miktarda paraduodenal sıvı birikimi oldu. Bu hasta konvansiyonel tedbirlerle 3 gün içerisinde iyileşti. Kanama, pankreatit veya açık perforasyon hiçbir hastada gelişmedi. Sonuç: Endoskopik sfinkterotomi sonrası 'large" ballon dilatasyonu, Billroth-II ameliyatı geçirmiş olan hastalarda zor safra yolu taşlarının çıkartılmasında etkili ve güvenli görülmektedir.
\end{abstract}

Anahtar kelimeler: Billroth II gastrektomi, large balon dilatasyon

\section{GİRISS}

Endoskopik sfinkterotomi (ES) safra yolu taşlarının çıkartılmasında en yaygın kullanılan tekniktir. Yalnız Billroth II gastrektomili (B-II) hastalarda standart endoskopik retrograt kolanjiopankreatikografi (ERCP) uygulamalarında farklılıklar bulunur. Papillaya ulaşmak için doğru endoskopi cihazı seçilmeli, afferent luptan geçilmeli ve duodenal güdüğe kadar girilmelidir. Papilla, B-II'de normal anatomik lokalizasyonuna göre 180 derece değişmiştir. Standart ERCP ile karşılaştırıldığında papilla ve biler aks ters pozisyondadır. Dolayısıyla bu anatomik değişiklikler ES'yi bu hastalarda zor ve tehlikeli hale getirmektedir. Bu nedenle yeni papillotomlar ve ES teknikleri geliştirilmeye çalışılmaktadır.
Background/aims: Patients with Billroth II gastrectomy, who have modified anatomy of the gastrointestinal tract, present technical difficulties during endoscopic stone removal. Recently, a large balloon dilatation has been used especially for extraction of difficult bile duct stones after endoscopic sphincterotomy. The aim of this study was to evaluate the efficacy and safety of endoscopic papillary large balloon dilatation with limited endoscopic sphincterotomy for removal of bile duct stones in patients with Billroth II gastrectomy. Methods: Twelve patients (12 men; median age: 69 years) with bile duct stones and a history of Billroth II gastrectomy were enrolled. After cannulation, limited endoscopic sphincterotomy was performed. Then, a large balloon dilatation (balloon size, 12-18 mm) was performed and stones were removed conventionally or via mechanical lithotripsy. Successful stone removal and complications were evaluated. Results: In all cases, stones were removed successfully. The median number of sessions for complete stone removal was one (range 1-3). Stone removal by mechanical lithotripsy was achieved in three patients (11.5\%). There were no significant complications such as bleeding, pancreatitis or perforation. Conclusions: Endoscopic papillary large balloon dilatation after endoscopic sphincterotomy appears to be an effective and safe method for removal of difficult bile duct stones in patients with Billroth II.

Key words: Billroth II gastrectomy, large balloon dilatation

Yakın zamanda yapılan çalışmalarda ES sonrası balon dilatasyonunun zor safra taşlarını çıkarmakta etkili olduğu gösterilmiştir (1-3). Bu metod B-II gastrektomili safra yolu taşı olan hastalarda kullanılabilir. Bu çalışmada safra yolu taşı olan B-II gastrektomili hastalarda kısmi ES ile birlikte büyük balon dilatasyonu'nun (LBD) etkinliği ve güvenliği değerlendirilmiştir.

\section{GEREÇ ve YÖNTEM}

Hastalar

Bu tek merkezli, prospektif bu çalışmaya Aralık 2009 ile Kasım 2010 tarihleri arasında Türkiye Yüksek Ihtisas Hastanesi 
Gastroenteroloji Kliniği'ne başvuran B-II gastrektomili ve safra yolu taşı olan hastalar dahil edildi. On iki [(hepsi erkek, ortalama yaş 69.0 (aralık: 48-84) yıl)] hastaya kısmi ES'yi takiben LBD ile taş çıkarma girişiminde bulunuldu.

Çalışmaya dahil edilme kriterleri, 18 yaşın üzerinde olmak ve safra yolu taşının 1 cm'den büyük olması idi. Çalışmadan dışlanma kriterleri ise safra yolu malignitesi şüphesi, kanama diatezi (INR $>1.5$, trombosit sayıs $<50.000 / \mathrm{mm}^{3}$, kanama zamanı >15 dakika), daha önce biliyer kanülasyon öyküsü ve sepsis idi.

Çalışma sonunda teknik başarı ve komplikasyonlar değerlendirildi. Komplikasyonlar konsensus guideline göre değerlendirildi (4). Çalışma için etik kurul onayı alındı.

\section{Teknik}

Üç deneyimli endoskopist hastalara ERCP yaptı. Sedasyon intravenöz midazolam ve meperidine ile sağlandı. Yan görüşlü duodeneskop kullanıldı. Selektif kanülasyonu takiben kısmi ES yapıldı. Kısmi ES; geleneksel ES'nin insizyon uzunluğunun yaklaşık 1/3'ü olarak tanımlandı. Kısmi ES'yi takiben skopi altında dilatator balon (Boston Scientific, ABD) yerleştirildi ve şişirildi. Balon çapı (12-18 mm) taş ve koledok çapına göre seçildi. Dilatasyon sonrası taşlar balon ve basket ile çıkartıldı. Balon ve basket ile çıkartılamayan hastalarda mekanik litotripsi (ML) uygulandi.

\section{BULGULAR}

On iki [hepsi erkek, ortalama yaş 69.0 (aralık: 48-84) yıl)] BII ameliyatı geçirmiş olan hastaya kısmi ES'yi takiben LBD ile taş çıkarma girişiminde bulunuldu. 6 hastaya $12 \mathrm{~mm}, 6$ hastaya $15 \mathrm{~mm}, 1$ hastaya 18 mm'lik balon ile dilatasyon yapıldı. Taş boyutu (enine çapı) ortalama 14,6 (aralık 12-18) mm idi. Koledok çapı ortalama 15,8 (aralık 10-21) mm idi. On bir hastanın taşları 1 seansta, 1 hastanın taşları 2 seansta çıkartıldı. Bir hastaya ML, 1 hastaya ESWL yardımı gerekti. Isslemden sonra 1 hastada az miktarda paraduodenal sıvı birikimi oldu. $\mathrm{Bu}$ hasta konvansiyonel tedbirlerle 3 gün içerisinde iyileşti. Hiçbir hastada kanama, pankreatit veya açık perforasyon gelişmedi (Tablo 1).

\section{TARTIŞMA}

B-II'li hastalarda papilin değişen anatomisi nedeniyle ERCP işlemi, ES ve taş çıkarma hem daha zor hem daha risklidir (5). $\mathrm{Bu}$ hastalarda işlemi zorlaştıran; afferent lupun entübasyonu, afferent lupta papillaya yaklaşma, değișen safra yolu kanülasyon yönüdür (6). Kanülasyon başarılı bir şekilde sağlansa bile değişmiş papilla anatomisi ES komplikasyonlarına yol açabilir (7-9). Ek olarak büyük, safra kanalı distaline impakte ve çok sayıdaki taşlar safra kanalından zor çıkartılırlar, bu nedenle zor taş olarak isimlendirilirler $(1,2)$. Gerek endoskopik olarak papile zor ulaşma ve gerek papilin anatomik değissik-

\begin{tabular}{|c|c|}
\hline Özellikler & \\
\hline Hasta sayısı & 12 \\
\hline Erkek/Kadın & $12 / 0$ \\
\hline Yaş (yll), ortalama (aralık) & $69(48-84)$ \\
\hline Taş boyutu (mm), ortalama (aralık) & $14,6(12-18)$ \\
\hline Koledok çapı (mm), ortalama (aralık) & $15,8(10-21)$ \\
\hline $\begin{array}{c}\text { Uygulanan ERCP seans1 } \\
1 \text { seans } \\
2 \text { seans }\end{array}$ & $\begin{array}{l}11 \text { hasta } \\
1 \text { hasta }\end{array}$ \\
\hline $\begin{array}{l}\text { Uygulanan balon büyüklügü } \\
\qquad \begin{array}{l}12 \mathrm{~mm} \\
15 \mathrm{~mm} \\
18 \mathrm{~mm}\end{array}\end{array}$ & $\begin{array}{l}6 \text { hasta } \\
6 \text { hasta } \\
1 \text { hasta }\end{array}$ \\
\hline ML ihtiyacı & 1 hasta \\
\hline $\begin{array}{l}\text { Komplikasyon } \\
\text { Paraduodenal sıvı birikimi } \\
\text { Perforasyon } \\
\text { Kanama } \\
\text { Pankreatit }\end{array}$ & $\begin{array}{l}1 \text { hasta } \\
- \\
- \\
-\end{array}$ \\
\hline
\end{tabular}

ML: Mekanik litotripsi

likleri nedeniyle B-II'li hastalarda ES daha az yapılmakta ve ES açıklığı az olduğundan büyük taşlar etkin olarak çıkartılamamaktadır (10). Bu zorlukları aşabilmek için B-II'li hastalarda çeşitli teknikler geliştirilmiştir. S-şeklinde sfinkteretom, B-II papillotom, stent rehberli iğne uçlu sfinkterotom bunlar içinde sayılabilir. Tüm bu tekniklerin B-II'li hastalarda safra taşı çıkartılmasındaki başarısı tatmin edici düzeyde değildir (911-12). Üstelik normal bireyler ile karşılaştırıldığında B-II'li hastalarda, ES ile hem kanama ve perforasyon sıklığı daha fazladır, hem de kanama olduğunda endoskopik kontrol daha zor olmaktadır (10-13-14).

Endoskopik balon dilatasyonu (EBD) taşların çıkartılmasında diğer bir alternatif bir yoldur (10). EBD göreceli olarak kolay bir uygulamadır ve ES ile karşılaştırıldığında kanama ve perforasyon riski daha azdır (10-15). Bununla beraber tek başına EBD'nunda ES'e göre daha küçük bir biliyer orifis sağlandığı için küçük taşlarda bile mekanik litotripsiye gerek duyulmakta veya işlem tekrarı gerekmektedir (16). EBD ile artan pankreatit riski de göz önüne alınırsa endoskopistler bu uygulamaya uzak durmaktadırlar (17).

Tüm bu zorluklar ve olumsuzlukların üstesinden gelmek üzere çeşitli yöntemler denenmiştir. 2003 yılından bu yana kısmi ES sonrası endoskopik papiller "large" balon dilatasyonu (EPLBD) safra yolu taşlarının çıkartılması için yaygın olarak kullanılmaya başlanmıştır (1-2-18-19). Kısmi ES'e EPLBD eklenmesi, daha geniş biliyer açıklık sağlamaktadır, taş çıkartılma başarısı ES veya EBD ile benzer oranlardadır (2-3). Yanı sıra bu yöntemle kanama ve pankreatit riski daha düşük bulunmuştur (1-2-17).

Literatürde taş çıkarmada kısmi ES'e EPLBD eklenmesi \%94 oranında başarılı ve \%4-5 gibi düşük komplikasyon oranı ile ilişkili bulunmuştur (18-19). Bizim çalışmamızda kısmi ES+ 
EPLBD ile tüm hastalarda başarılı taş ekstraksiyonu sağlanırken; kanama, pankreatit ve açık perforasyon gibi bir komplikasyon hiçbir hastada görülmedi. Sadece bir hastada işlemden sonra az miktarda paraduodenal sıvı birikimi oldu. Bu hasta konvansiyonel tedbirler ile 3 gün içerisinde iyileşti.

Kısmi ES'e EBD eklenmesi ve tek başına ES'i karşılaştıran bir çalışmada, B-II gastrektomili hastalarda taş çıkartılmasında tek başına ES'de daha düşük başarı (\%83 vs \%100) ve yüksek komplikasyon riskinin yanı sıra artmış kanama riski (\%17) saptanmıştır. Bergmann ve ark. yapmış olduğu ES ve kısmi ES+EPLBD'i karşılaştıran çalışmada da; ES'in daha yüksek oranda mekanik litotripsi gerektirdiğini saptamıştır (\%22 vs \%11.5). Buna göre kismi ES+EPLBD, B-II'li hastalarda daha başarılı ve güvenli gibi görünmektedir. Öte yandan EBD daha düşük başarı, daha yüksek komplikasyon oranı ile ilişkili bulunmuş, EBD'nunda ML gerekliliğii, EPLBD'e göre daha yüksek oranda saptanmıştır (\%19-\%11.5) (10). Bizim çalışmamızda sadece bir hastaya ML yapılması gerekmiştir (\%8).

Itoi ile Kim, çalışmalarında ES+EPLBD ile başarılı taş çıkarma sonuçlarını \%89 ve \%100 olarak saptamışlar ve hiç belirgin komplikasyon izlememişler $(22,23)$. Choi ve ark. çalışmalarında hastalarda taş çıkartılması için 2 yeni metod geliştirmişlerdir. Bunlar dönebilen papillotom ve biliyer stent rehberliğinde iğne uçlu sfinkteretomdur. Her iki metodla yapılan ES sonuçları arasında taş çıkartılma başarısı ve komplikasyonlar açısından bir fark bulunmamıştır (24). Bizim çalışmamızda taş çıkarma başarı oranımız \%100 idi. 1 hastaya ML ve 1 hastaya da ESWL yardımı gerekti. Seans sayısı l'den fazla olan hastada da taşlar 2. seansta çıkartıldı. Hiçbir hastaya taş çıkarılamaması nedeniyle perkütan girişim ve cerrahi uygulanmadı.

EPLBD uygulamasının potansiyel riski perforasyondur (25, 26). B-II gastrektomili hastalarda perforasyon geliştiğinde cerrahi onarım normal anatomili bireylere göre daha zor olmakta ve mortalite oranı daha yüksek seyretmektedir. Bu nedenle çalışmamızda dilatasyon balonu çapını belirlerken koledok çapını göz önüne aldık ve en fazla 18 mm'lik balon kullandık. Choi'nin çalışmasında dilatasyon balon çapı en fazla $15 \mathrm{~mm}$ ile sinırlı tutulmuş, sonra balon çapının 10-20 mm olduğu Itoi'nin çalışması ile karşılaştırma yapılmış. Her iki çalışmada taş çıkartılma oranı benzer bulunmuş ve ML ihtiyacında artış saptanmamış (24). Dilatasyon balonu çapının seçimi koledok çapı ve duruma göre taşın çapı göz önüne alınarak endoskopistin deneyimine göre belirlenmelidir.

Sonuç olarak; Billroth II'li hastalarda biliyer taş çıkartılması için standart bir teknik yoktur. B-II'li hastalarda geleneksel ES ile karşılaştırıldığında kısmi ES teknik olarak daha kolaydır. Kısmi ES'e EPLBD eklendiğinde biliyer açıklık genişlemekte ve büyük safra taşlarının geçişine izin vermektedir. Böylece işlem esnasında ML'ye daha az ihtiyaç duyulmaktadır. Komplikasyon açısından tek başına ES veya EBD'a göre daha az komplikasyon oranına sahiptir. B-II'li hastalarda kısmi ES+EPLBD etkili ve güvenli bir teknik gibi görünmektedir.

\section{KAYNAKLAR}

1. Ersöz G, Tekeşin O, Özütemiz AO, Günşar, F. Biliary sphincterotomy plus dilation with a large balloon for bile duct stones that are difficult to extract. Gastrointest Endosc 2003;57:156-9.

2. Heo JH, Kang DH, Jung HJ, et al. Endoscopic sphincterotomy plus large-balloon dilation versus endoscopic sphincterotomy for removal of bile-duct stones. Gastrointest Endosc 2007;66:720-6

3. Itoi T, Itokawa F, Sofuni A, et al. Endoscopic sphincterotomy combined with large balloon dilation can reduce the procedure time and fluoroscopy time for removal of large bile duct stones. Am J Gastroenterol 2009; 104:560-5.

4. Cotton PB, Lehman G, Vennes J, et al. Endoscopic sphincterotomy complications and their management: an attempt at consensus. Gastrointest Endosc 1991;37:383-93.

5. Forbes A, Cotton PB. ERCP and sphincterotomy after Billroth II gastrectomy. Gut 1984;25:971-4

6. Hintze RE, Adler A, Veltzke W, Abou-Rebyeh H. Endoscopic access to the papilla of Vater for endoscopic retrograde cholangiopancreatography in patients with Billroth II or Roux-en-Y gastrojejunostomy. Endoscopy 1997;29:69-73

7. Safrany L, Neuhaus B, Portocarrero G, Krause S. Endoscopic sphincterotomy in patients with Billroth II gastrectomy. Endoscopy 1980;12:1622

8. Rosseland AR, Osnes M, Kruse A. Endoscopic sphincterotomy (EST) in patients with Billroth II gastrectomy. Endoscopy 1981;13:19-24.

9. Hintze RE, Veltzke W, Adler A, Abou-Rebyeh H. Endoscopic sphincterotomy using an S-shaped shincterotome in patient with a Billroth II or Roux-en-Y gastrojejunostomy. Endoscopy 1997; 29:74-8.

10. Bergman JJ, van Berkel AM, Bruno MJ, et al. A randomized trial of endoscopic balloon dilation and endoscopic sphincterotomy for removal of bile duct stones in patients with a prior Billroth II gastrectomy. Gastrointest Endosc 2001;53:19-26.

11. Wang YG, Binmoeller KF, Seifert H, et al. A new guide wire papillotome for patients with Billroth II gastrectomy. Endoscopy 1996; 28:254-5.

12. Al-Kawas FH, Geller AJ. A new approach to sphincterotomy in patients with Billroth II gastrectomy. Gastrointest Endosc 1996;43:253-5.

13. Faylona JM, Qadir A, Chan AC, et al. Small-bowel perforations related to endoscopic retrograde cholangiopancreatography (ERCP) in patients with Billroth II gastectomy. Endoscopy 1999;31:546-9.

14. Kim MH, Lee SK, Lee MH, et al. Endoscopic retrograde cholangiopancreatography and needle-knife sphincterotomy in patients with Billroth II gastrectomy: a comparative study of the forward-viewing endoscope and the side-viewing duodenoscope. Endoscopy 1997;29:82-5.

15. Prat F, Fritsch J, Choury AD, et al. Endoscopic sphincteroplasty: a useful therapeutic tool for biliary endoscopy in Billroth II gastrectomy patients. Endoscopy 1997;29:79-81

16. Bergman JJ, Rauws EA, Fockens P, et al. Randomized trial of endoscopic balloon dilatation versus endoscopic sphincterotomy for removal of bile duct stones. Lancet 1997;349:1124-9.

17. Disario JA, Freeman ML, Bjorkman DJ, et al. Endoscopic balloon dilatation compared with sphincterotomy for extraction of bile duct stones. Gastroenterology 2004;127:1291-9.

18. Yoo BM, Kim JH, Jung JH, et al. Large balloon sphincteroplasty along with or without sphincterotomy in patients with large extrahepatic bile duct stones: multi center study. Gastrointest Endosc 2007;65:AB97. 
19. Maydeo A, Bhandari S. Balloon sphincteroplasty for removing difficult bile duct stones. Endoscopy 2007;39:958-61.

20. Nakahara K, Horaguchi J, Fujita N, et al. Therapeutic endoscopic retrograde cholangiopancreatography using an anterior oblique-viewing endoscope for bile duct stones in patients with prior Billroth II gastrectomy. J Gastroenterol 2009;44:212-7.

21. van Buuren HR, Boender J, Nix GA, van Blankenstein M. Needle-knife sphincterotomy guided by a biliary endoprosthesis in Billroth II gastrectomy patients. Endoscopy 1995;27:229-32.

22. Itoi $\mathrm{T}$, Ishii $\mathrm{K}$, Itokawa $\mathrm{F}$, et al. Large balloon papillary dilation for removal of bile duct stones in patients who have undergone a Billroth II gastrectomy. Dig Endosc. 2010;22:S98-102.
23. Kim GH, Kang DH, Song GA, et al. Endoscopic removal of bile-duct stones by using a rotatable papillotome and a large-balloon dilator in patients with a Billroth II gastrectomy. Gastrointest Endosc 2008;67:1134-8.

24. Choi CW, Choi JS, Kang DH, et al. Endoscopic papillary large balloon dilation in Billroth II gastrectomy patients with bile duct stones. J Gastroenterol Hepatol 2011 Jul 28. doi: 10.1111/j.1440-1746.2011.06863.

25. Park SY, Park CH, Yoon KW, et al. Endoscopic large-diameter balloon dilation after fistulotomy for the removal of bile duct stones in a difficult cannulation. Gastrointest Endosc. 2009;69:955-9.

26. Attasaranya S, Cheon YK, Vittal H, et al. Large-diameter biliary orifice balloon dilation to aid in endoscopic bile duct stone removal: a multicenter series. Gastrointest Endosc. 2008;67:1046-52. 